\title{
Characterization of proteoforms with unknown post-translational modifications using the MIScore (Supporting Information)
}

\author{
Qiang $\mathrm{Kou}^{1}$, Binhai Zhu ${ }^{2}$, Si $\mathrm{Wu}^{3}$, Charles Ansong ${ }^{4}$, Nikola Tolić ${ }^{5}$, Ljiljana Paša-Tolić ${ }^{5}$, \\ and Xiaowen $\mathrm{Liu}^{1,6}$ \\ ${ }^{1}$ Department of BioHealth Informatics, Indiana University-Purdue University Indianapolis \\ ${ }^{2}$ Department of Computer Science, Montana State University \\ ${ }^{3}$ Department of Chemistry and Biochemistry, Univeristy of Oklahoma \\ ${ }^{4}$ Biological Sciences Division, Pacific Northwest National Laboratory \\ ${ }^{5}$ Environmental Molecular Sciences Laboratory, Pacific Northwest National Laboratory \\ ${ }^{6}$ Center for Computational Biology and Bioinformatics, Indiana University School of \\ Medicine
}




\section{Contents}

1 Abbreviations $\quad$ S1

2 Computation of the distribution of shared mass count scores between $S$ and proteoforms in $\mathcal{Q}_{i}$

3 Computation of the distribution of shared mass count scores between $S$ and proteoforms in $\mathcal{Q}_{i j}$

\section{List of Figures}

S1 An algorithm for computing the distribution of shared mass counts between a spectrum and the proteoforms of a protein with a pair of ordered modifications. . . . . . . . . . . . S2

S2 An algorithm for computing the distribution of shared mass count scores between spectrum

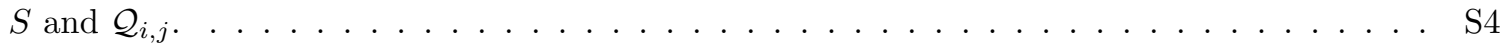

S3 Comparison of the accuracy of the model for determining the number of modifications with different settings $0.5,0.6, \ldots, 1,1.1, \ldots, 2$ of the ratio $r \ldots \ldots \ldots \ldots \ldots \ldots$

S4 Percentages of identified modification sites and correctly identified ones by the MIScore method from the 6100 PrSMs with one modification with various MIScore cutoff values. . . S6

S5 Percentages of identified modification sites and correctly identified ones by the MIScore method from the 6100 PrSMs with a truncation at the $\mathrm{N}$ (or C) terminus and one PTM near the $\mathrm{N}$ (or $\mathrm{C}$ ) terminus with various MIScore cutoff values. . . . . . . . . . . . . . S S7

S6 The modification sites reported by the MIScore method from the 6100 PrSMs with a truncation at the $\mathrm{N}$ ( or $\mathrm{C}$ ) terminus and one PTM near the $\mathrm{N}$ (or $\mathrm{C}$ ) terminus are grouped into bins with width 0.1 based on their modification identification scores. The average identification score and accuracy rate of the modification sites in each bin are compared. . . . . . . . . . . S8

S7 The ordered modification pairs reported by the MIScore method from the 6100 PrSMs with two modifications are grouped into bins with width 0.1 based on their confidence scores. The average confidence score and accuracy rate of the ordered modification pairs in each bin are

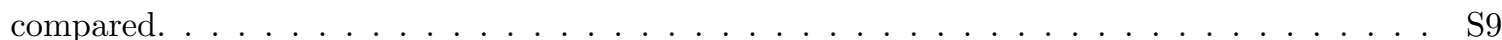

S8 Percentages of identified modification sites and correctly identified ones by the MIScore method from the $6100 \mathrm{PrSMs}$ with two modifications with various MIScore cutoff values. . . S10 
S9 The modification sites reported by the MIScore method from the 6100 PrSMs with two modification are grouped into bins with width 0.1 based on their modification identification scores. The average identification score and accuracy rate of the modification sites in each bin are compared. . . . . . . . . . . . . . . . . . . . . . . . . S11

\section{List of Tables}

S1 Parameters used in TopPIC . . . . . . . . . . . . . . . . . . . S11

S3 Probabilities estimated from the 305 training PrSMs of the EC data set . . . . . . . . . S12

S5 Parameters used in Mascot for searching the 38 top-down MS/MS spectra against the SwissProt EC proteome database. . . . . . . . . . . . . . . . . . . . . . . S12

S9 Parameters used in MS-GF $+\ldots \ldots \ldots \ldots \ldots \ldots \ldots$. . . . . . . . . . . . . . . . . . . . .

S10 A total of 8 cysteinylation sites reported from ST data set that are supported peptides identified from the bottom-up data set by MS-GF+ . . . . . . . . . . . . . S13 


\section{Abbreviations}

CID: Collision-induced dissociation

EC: $\quad$ Escherichia coli

FDR: $\quad$ False discovery rate

HCD: Higher energy collisional dissociation

HPLC: High-performance liquid chromatography

LPM: $\quad$ Low-pH minimal medium

MIScore: Modification Identification Score

MS: Mass spectrometry

MS/MS: Tandem mass spectrometry

PrSM: Proteoform-spectrum match

PSA: $\quad$ Primary structure alteration

PTM: Post-translational modification

RPLC: Reversed phase liquid chromatography

ST: $\quad$ Salmonella typhimurium

\section{Computation of the distribution of shared mass count scores between $S$ and proteoforms in $\mathcal{Q}_{i}$}

We fill out a three-dimensional table $D(f, g, h)$ for $f=0,1$, and 2 . We use the following recurrence functions to compute the values $D(f, g, h)$ that are not initialized:

$$
\begin{gathered}
D(0, g, h)= \begin{cases}D\left(0, g-1, h-s_{0, g}\right) & \text { if } h \geq s_{0, g} ; \\
0 & \text { otherwise. }\end{cases} \\
D(1, g, h)= \begin{cases}D\left(0, g-1, h-s_{1, g}\right)+D\left(1, g-1, h-s_{1, g}\right) & \text { if the } g \text { th amino acid is a modification } \\
D\left(1, g-1, h-s_{1, g}\right) & \text { site of } x_{i} \text { and } h \geq s_{1, g} ; \\
0 & \text { if the } g \text { th amino acid cannot be modified } \\
\text { by } x_{i} \text { and } h \geq s_{1, g} ;\end{cases} \\
D(2, g, h)= \begin{cases}D\left(1, g-1, h-s_{2, g}\right)+D\left(2, g-1, h-s_{2, g}\right) & \text { if the } g \text { th amino acid is a modification } \\
D\left(2, g-1, h-s_{2, g}\right) & \text { site of } y_{i} \text { and } h \geq s_{2, g} ; \\
0 & \text { if the } g \text { th amino acid is not a modification } \\
\text { site of } y_{i} \text { and } h \geq s_{2, g} ; & \text { otherwise. }\end{cases}
\end{gathered}
$$




\section{Algorithm}

Input: A spectrum $S$ with $n$ neutral fragment masses, a unmodified protein sequence $P$ with length $m$, and a pair of ordered modifications $\left(x_{i}, y_{i}\right)$. The precursor mass of $S$ matches the sum of the molecule mass of protein $P$ and the masses of $x_{i}$ and $y_{i}$.

Output: The distribution of the shared mass counts between $S$ and the proteoforms of $P$ with the ordered modifications $\left(x_{i}, y_{i}\right)$.

1. Compute $s_{f, g}$ for $f=0,1,2$ and $g=1,2, \ldots, m$. Set $t=\min \{n, 2 m-2\}$ as the maximum possible shared mass count.

2. Set $D(0,0,0)=1$, set $D(0,0, h)=0$ for $1 \leq h \leq t$, set $D(1,0, h)=0$ for $0 \leq h \leq t$, and set $D(2,0, h)=0$ for $0 \leq h \leq t$.

3. For $g=1$ to $m$ do

4. For $h=0$ to $t$ do

5. If $h \geq s_{0, g}$ then $D(0, g, h)=D\left(0, g-1, h-s_{0, g}\right)$ else $D(0, g, h)=0$

6. If $h \geq s_{1, g}$ then

7. If the $g$ th amino acid is a modification site of $x_{i}$ then

8. $D(1, g, h)=D\left(0, g-1, h-s_{1, g}\right)+D\left(1, g-1, h-s_{1, g}\right)$

9. $\quad$ else

10. $\quad D(1, g, h)=D\left(0, g-1, h-s_{1, g}\right)$

11. else

12. $D(1, g, h)=0$

13. If $h \geq s_{2, g}$ then

14. If the $g$ th amino acid is a modification site of $y_{i}$ then

15. $D(2, g, h)=D\left(1, g-1, h-s_{2, g}\right)+D\left(2, g-1, h-s_{2, g}\right)$

16. else

17. $D(2, g, h)=D\left(1, g-1, h-s_{2, g}\right)$

18. else

19. $\quad D(2, g, h)=0$

20 . Report the values $D(2, m, h)$ for $0 \leq h \leq t$.

Figure S1: An algorithm for computing the distribution of shared mass counts between a spectrum and the proteoforms of a protein with a pair of ordered modifications. 
The methods for identifying two modifications from a mass shift can be extended to multiple modifications. When a mass shift results from $K$ modifications, the dynamic programming algorithm in Figure S1 is modified to fill out a table $D(f, g, h)$ for $f=0,1, \ldots, K$. We extend the definitions of $B_{f, g}$ and $s_{f, g}$ for $f=3,4, \ldots, K$ and fill out the table using the following recurrence function:

$$
D(f, g, h)= \begin{cases}D\left(f, g-1, h-s_{f, g}\right)+D\left(2, g-1, h-s_{f, g}\right) & \text { if the } g \text { th amino acid is a site of } \\ & \text { the } f \text { th modification and } h \geq s_{f, g} ; \\ D\left(2, g-1, h-s_{f, g}\right) & \text { if the } g \text { th amino acid is not a site of } \\ & \text { the } f \text { th modification and } h \geq s_{f, g} ; \\ 0 & \text { otherwise. }\end{cases}
$$

\section{Computation of the distribution of shared mass count scores between $S$ and proteoforms in $\mathcal{Q}_{i j}$}

Similar to the algorithm in Figure S1, we fill out a three dimensional table $D(f, g, h)$. The initialization step is the same to Equation (4) in the paper. The recurrence functions to fill out the table are

$$
\begin{aligned}
& D(0, g, h)= \begin{cases}D\left(0, g-1, h-s_{0, g}\right) & \begin{array}{l}
\text { if } h \geq s_{0, g} ; \\
0
\end{array} \\
\text { otherwise. }\end{cases} \\
& D(1, g, h)= \begin{cases}D\left(0, g-1, h-s_{1, g}\right)+D\left(1, g-1, h-s_{1, g}\right) & \text { if the } g \text { th amino acid is a modification; } \\
D\left(1, g-1, h-s_{1, g}\right) & \text { site of } x_{i} \text { and } h \geq s_{1, g} \text { and } g \leq j ; \\
0 & \text { if the } g \text { th amino acid cannot be modified }\end{cases} \\
& D(2, g, h)= \begin{cases}D\left(1, g-1, h-s_{2, g}\right)+D\left(2, g-1, h-s_{2, g}\right) & \text { if the } g \text { th amino acid is a modification } h \geq s_{1, g} ; \\
D\left(2, g-1, h-s_{2, g}\right) & \text { site of } y_{i} \text { and } h \geq s_{2, g} \text { and } g>j ; \\
0 & \text { if the } g \text { th amino acid is not a modification }\end{cases} \\
& \text { site of } y_{i} \text { and } h \geq s_{2, g} ; \\
&
\end{aligned}
$$




\section{Algorithm}

Input: A spectrum $S$ with $n$ neutral fragment masses, a unmodified protein sequence $P$ with length $m$, a pair of ordered modifications $\left(x_{i}, y_{i}\right)$, and a number $j$. The precursor mass of $S$ matches the sum of the molecule mass of protein $P$ and the masses of $x_{i}$ and $y_{i}$.

Output: The distribution of the shared mass counts between $S$ and the proteoforms in $\mathcal{Q}_{i, j}$.

1. Compute $s_{f, g}$ for $f=0,1,2$ and $g=1,2, \ldots, m$. Set $t=\min \{n, 2 m-2\}$ as the maximum possible shared mass count.

2. Set $D(0,0,0)=1$, set $D(0,0, h)=0$ for $1 \leq h \leq t$, set $D(1,0, h)=0$ for $0 \leq h \leq t$, and set $D(2,0, h)=0$ for $0 \leq h \leq t$.

3. For $g=1$ to $m$ do

4. For $h=0$ to $t$ do

5. If $h \geq s_{0, g}$ then $D(0, g, h)=D\left(0, g-1, h-s_{0, g}\right)$ else $D(0, g, h)=0$

6. If $h \geq s_{1, g}$ then

7. If the $g$ th amino acid is a modification site of $x_{i}$ and $g \leq j$ then

8. $\quad D(1, g, h)=D\left(0, g-1, h-s_{1, g}\right)+D\left(1, g-1, h-s_{1, g}\right)$

9. $\quad$ else

10. $\quad D(1, g, h)=D\left(0, g-1, h-s_{1, g}\right)$

11. else

12. $D(1, g, h)=0$

13. If $h \geq s_{2, g}$ then

14. If the $g$ th amino acid is a modification site of $y_{i}$ and $g>j$ then

15. $D(2, g, h)=D\left(1, g-1, h-s_{2, g}\right)+D\left(2, g-1, h-s_{2, g}\right)$

16. else

17. $D(2, g, h)=D\left(1, g-1, h-s_{2, g}\right)$

18. else

19. $\quad D(2, g, h)=0$

20 . Report the values $D(2, m, h)$ for $0 \leq h \leq t$.

Figure S2: An algorithm for computing the distribution of shared mass count scores between spectrum $S$ and $\mathcal{Q}_{i, j}$. 


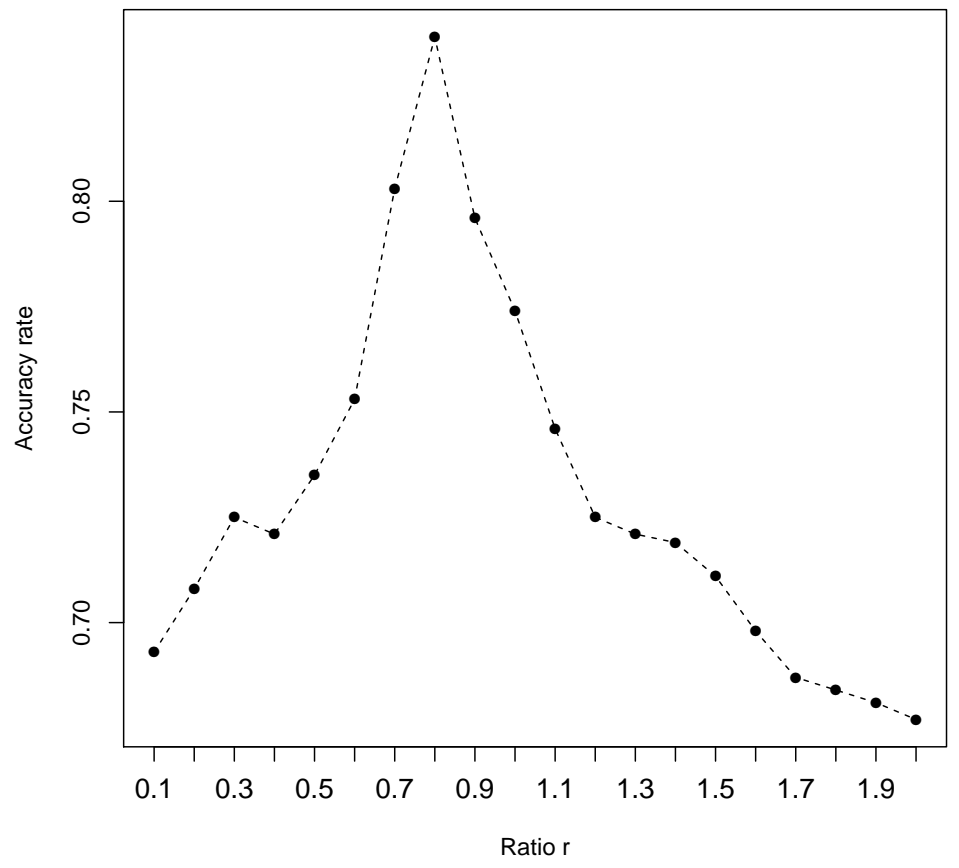

Figure S3: Comparison of the accuracy of the model for determining the number of modifications with different settings $0.5,0.6, \ldots, 1,1.1, \ldots, 2$ of the ratio $r$. 


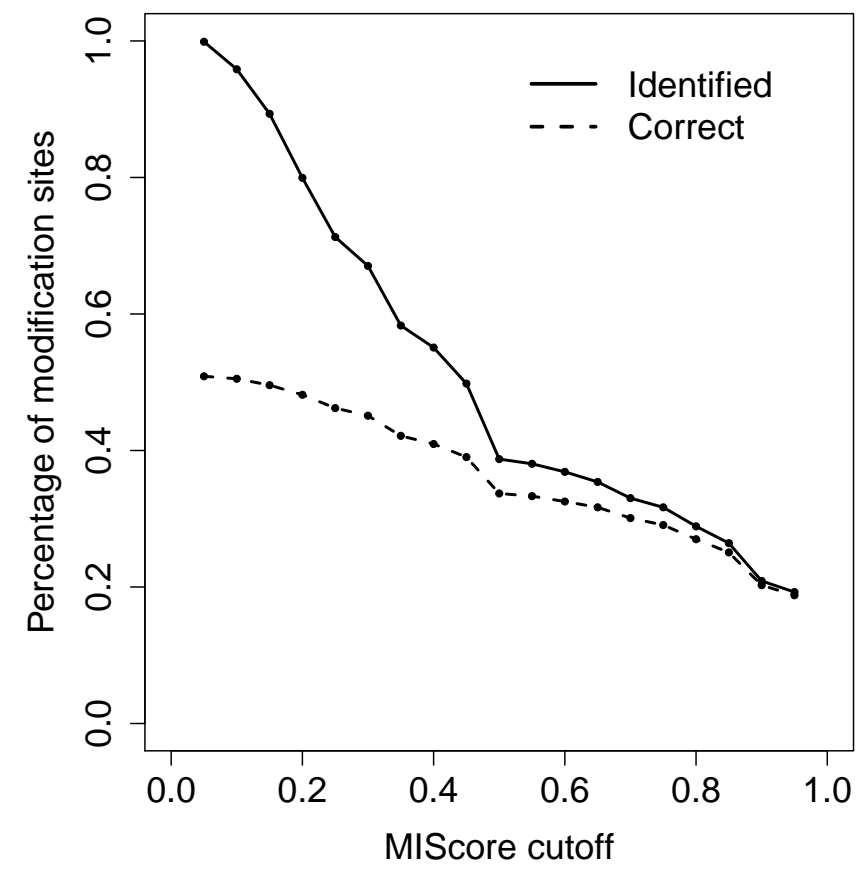

Figure S4: Percentages of identified modification sites and correctly identified ones by the MIScore method from the 6100 PrSMs with one modification with various MIScore cutoff values. 


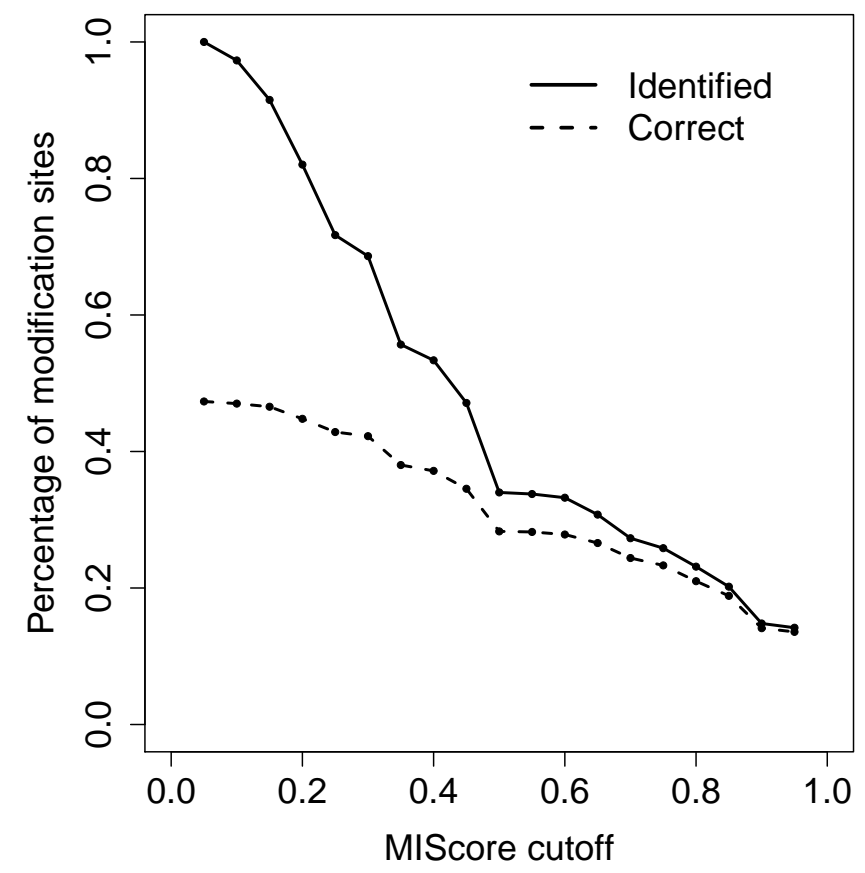

Figure S5: Percentages of identified modification sites and correctly identified ones by the MIScore method from the 6100 PrSMs with a truncation at the $\mathrm{N}$ (or C) terminus and one PTM near the $\mathrm{N}$ (or $\mathrm{C}$ ) terminus with various MIScore cutoff values. 


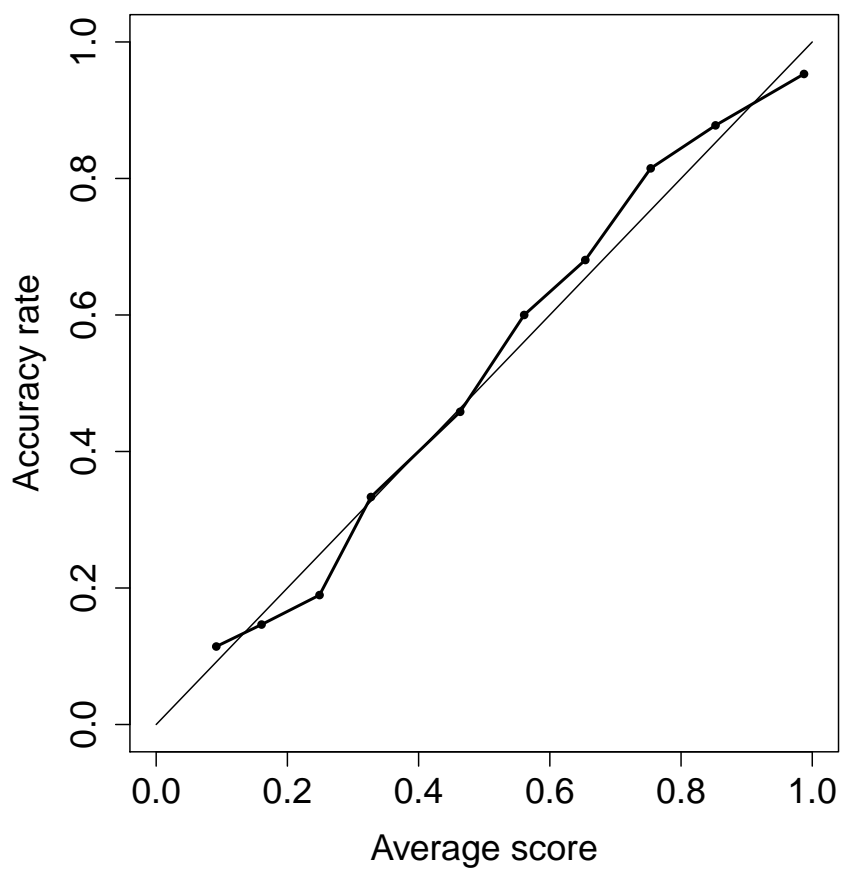

Figure S6: The modification sites reported by the MIScore method from the 6100 PrSMs with a truncation at the $\mathrm{N}$ (or $\mathrm{C}$ ) terminus and one PTM near the $\mathrm{N}$ (or C) terminus are grouped into bins with width 0.1 based on their modification identification scores. The average identification score and accuracy rate of the modification sites in each bin are compared. 


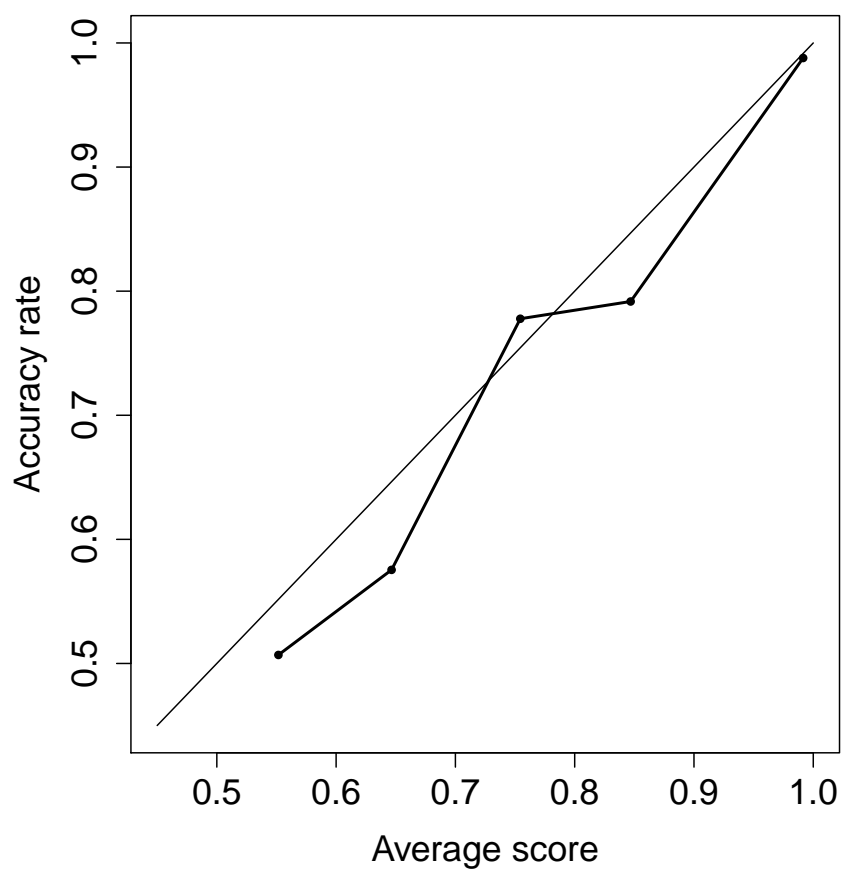

Figure S7: The ordered modification pairs reported by the MIScore method from the 6100 PrSMs with two modifications are grouped into bins with width 0.1 based on their confidence scores. The average confidence score and accuracy rate of the ordered modification pairs in each bin are compared. 


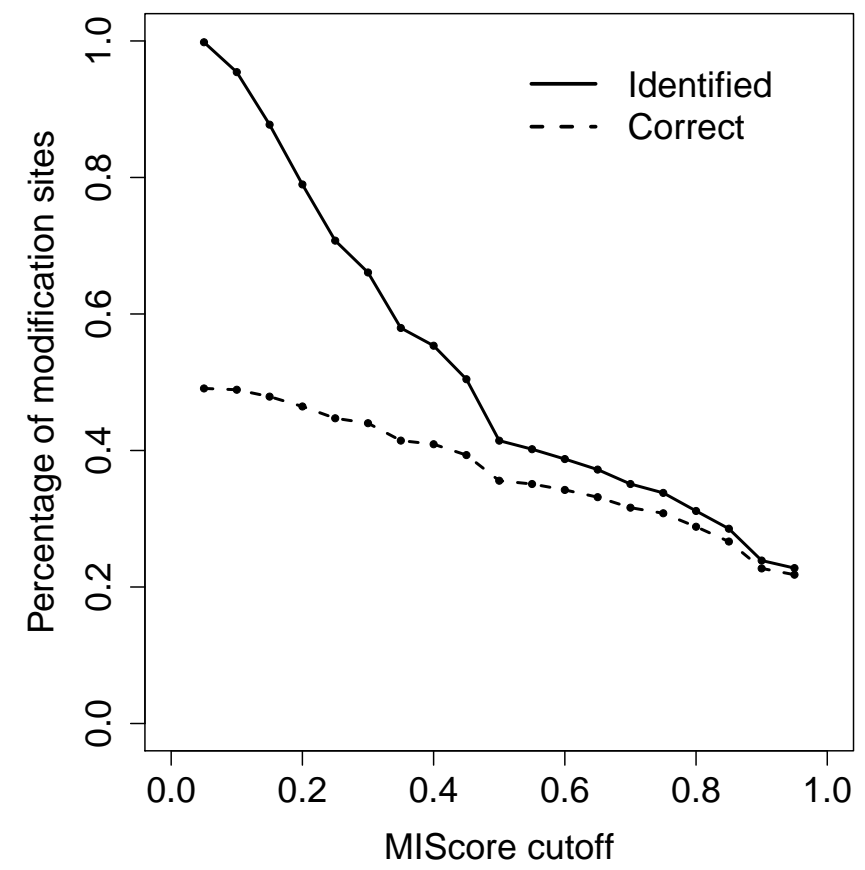

Figure S8: Percentages of identified modification sites and correctly identified ones by the MIScore method from the 6100 PrSMs with two modifications with various MIScore cutoff values. 


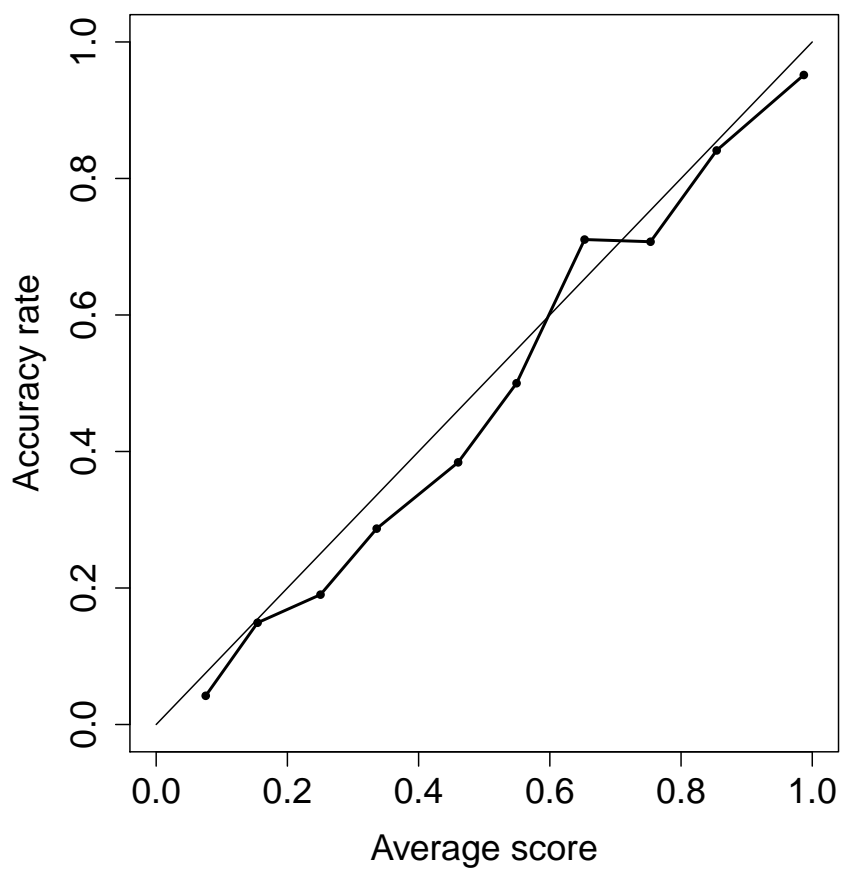

Figure S9: The modification sites reported by the MIScore method from the 6100 PrSMs with two modification are grouped into bins with width 0.1 based on their modification identification scores. The average identification score and accuracy rate of the modification sites in each bin are compared.

\begin{tabular}{cc}
\hline Parameter & Value \\
\hline Cysteine protecting group & None \\
Using a decoy protein database & True \\
Error tolerance & $15 \mathrm{ppm}$ \\
Maximum absolute value of unexpected mass shifts & $300 \mathrm{Da}$ \\
Maximum number of unexpected mass shifts & 2 \\
Cutoff type & FDR \\
Cutoff value & 0.01 \\
\hline
\end{tabular}

Table S1: Parameters used in TopPIC 


\begin{tabular}{cc}
\hline Probability & Value \\
\hline $\operatorname{Pr}\left(X_{1}=1\right)$ & 0.0886 \\
$\operatorname{Pr}\left(X_{1}=0\right)$ & 0.9114 \\
$\operatorname{Pr}\left(X_{0}=1\right)$ & 0.0042 \\
$\operatorname{Pr}\left(X_{0}=0\right)$ & 0.9958 \\
\hline
\end{tabular}

Table S3: Probabilities estimated from the 305 training PrSMs of the EC data set

\begin{tabular}{cc}
\hline Parameter & Value \\
\hline Database & SwissProt \\
Enzyme & No cleavage \\
Taxonomy & E.coli \\
Peptide error tolerance & $\pm 15 \mathrm{ppm}$ \\
MS/MS error tolerance & $\pm 15 \mathrm{ppm}$ \\
Peptide charge & $1+$ \\
Mass values & Monoisotopic \\
\hline
\end{tabular}

Table S5: Parameters used in Mascot for searching the 38 top-down MS/MS spectra against the Swiss-Prot EC proteome database.

\begin{tabular}{cc}
\hline Parameter & Value \\
\hline Precursor mass tolerance & $20.0 \mathrm{ppm}$ \\
Isotope error tolerance & 0,1 \\
Target-decoy analysis & True \\
Instrument & High resolution \\
Enzyme & Trypsin \\
Protocol & Standard \\
Number of tolerable termini & 0 \\
Minimum peptide length & 6 \\
Maximum peptide length & 40 \\
Number of matches per spectrum & 1 \\
\hline
\end{tabular}

Table S9: Parameters used in MS-GF+ 


\begin{tabular}{ccc}
\hline UniProt ID & Description & Modification site \\
\hline A0A0F6B9X6 & Elongation factor Tu & C256 \\
A0A0F6B6G9 & Phosphoglycerate kinase & C249 \\
A0A0F6BAJ3 & 60 kDa chaperonin & C138 \\
A0A0F6BAJ3 & 60 kDa chaperonin & C458 \\
A0A0F6B221 & Putative cytoplasmic protein & C75 \\
A0A0F6B244 & DNA-binding protein & C21 \\
A0A0F6B623 & Enolase & C247 \\
A0A0F6BAI0 & Acid phosphatase & C194 \\
\hline
\end{tabular}

Table S10: A total of 8 cysteinylation sites reported from ST data set that are supported peptides identified from the bottom-up data set by MS-GF+. 